PHYSICAL REVIEW B 82, 165443 (2010)

\title{
Stability and dynamics of small molecules trapped on graphene
}

\author{
Rolf Erni* \\ Electron Microscopy Center, Swiss Federal Laboratories for Materials Science and Technology, EMPA, CH-8600 Dübendorf, Switzerland \\ Marta D. Rossell \\ Department of Materials, ETH Zürich, CH-8093 Zürich, Switzerland
}

Manh-Thuong Nguyen, Stephan Blankenburg, and Daniele Passerone

Swiss Federal Laboratories for Materials Science and Technology, nanotech@surfaces laboratory, EMPA, CH-8600 Dübendorf, Switzerland

Peter Hartel

CEOS GmbH, D-69126 Heidelberg, Germany

\author{
Nasim Alem, Kris Erickson, Will Gannett, and Alex Zettl \\ Department of Physics, University of California at Berkeley, Berkeley, California 94720, USA \\ and Materials Sciences Division, Lawrence Berkeley National Laboratory, Berkeley, California 94720, USA
}

(Received 1 October 2010; published 25 October 2010)

\begin{abstract}
Chromatic and spherical aberration-corrected atomic-resolution transmission electron microscopy combined with density-functional theory calculations is employed to elucidate the stability and dynamics of admolecules on suspended graphene. The results presented provide evidence that the interaction between the molecules and hydrogen adatoms leads to a mutual trapping. These "symbiotic" configurations can explain the presence of stable admolecules on graphene at ambient temperature. It is proposed to exploit these configurations to functionalize and dope graphene.
\end{abstract}

DOI: 10.1103/PhysRevB.82.165443

PACS number(s): 81.05.ue, 31.15.A-, 68.37.Og

\section{INTRODUCTION}

Graphene, a sheet of hexagonally arranged carbon atoms, ${ }^{1-3}$ is one of the top candidates of materials on which future electronics shall be built upon. Knowledge about its properties and any likely occurring modification is of essential importance considering its implementation in devices. Whether, how, and particularly, which local defects can occur and explain graphene's measurable physical properties, as, for instance, the limited charge-carrier mobility, is under debate. Moreover, tailoring the properties of a material primarily requires an understanding on how the material can be modified in a controlled manner. In order to modify graphene's pristine properties, it can be decorated by molecules or adatoms. ${ }^{4-8}$ Such attachments can be considered as defects and act as electronically active dopants which control the electronic structure of graphene and its conductivity. Indeed, the importance of such local modifications is reflected in graphene's measurable properties. As the carrier mobility of graphene is lower than the value predicted by theory, it is suspected that defects, such as adatoms, admolecules or vacancies, account for this discrepancy. ${ }^{9}, 10$ Yet, adhesion and migration energies reported for many elements and molecules would make them largely mobile at room temperature. .,7,, 11 This, of course, complicates to explain their effect in a static picture. Here we present a combined experimental and theoretical study of small molecules on graphene which provides evidence that by forming energetically favorable configurations, small molecules can be stable on graphene at room temperature.

\section{METHODS}

\section{A. Experiment}

Freestanding suspended monolayer graphene membranes ${ }^{12,13}$ were used for atomic-resolution transmission electron microscopy (TEM) employing a spherical and chromatic aberration-corrected microscope. ${ }^{14,15}$ The quadrupoleoctupole-type $C_{C} / C_{S}$ corrector consists of ten quadrupole stages. Two of them produce crossed electromagnetic quadrupole fields for the correction of the chromatic aberration $C_{C}$ and octupole fields for the correction of the third-order spherical aberration $C_{S}\left(=C_{3}\right)$. All axial aberrations up to fourth order and all axial chromatic aberrations of first degree up to first order can be compensated semiautomatically. The fifth-order aberrations are by design sufficiently small to allow for a spatial resolution of about $80 \mathrm{pm}$ at $80 \mathrm{kV}$. As the effect of $C_{C}$ was annulled by the aberration corrector, in contrast to earlier experiments, ${ }^{12,16}$ no monochromator was necessary to achieve a sufficiently high information limit at $80 \mathrm{kV}$.

In order to derive direct structural and qualitative chemical information about the nature of the adatoms, focal series of atomic-resolution micrographs were recorded which were processed to retrieve the complex electron wave at the exit plane of the specimen. This step unravels the microscope's transfer function affecting individual micrographs and restores phase and amplitude of the diffracted electron wave. ${ }^{17-20}$ Yet the result of a reconstruction represents an average of the structure over the period of time the series is recorded. Therefore, in addition to the data retrieved from 


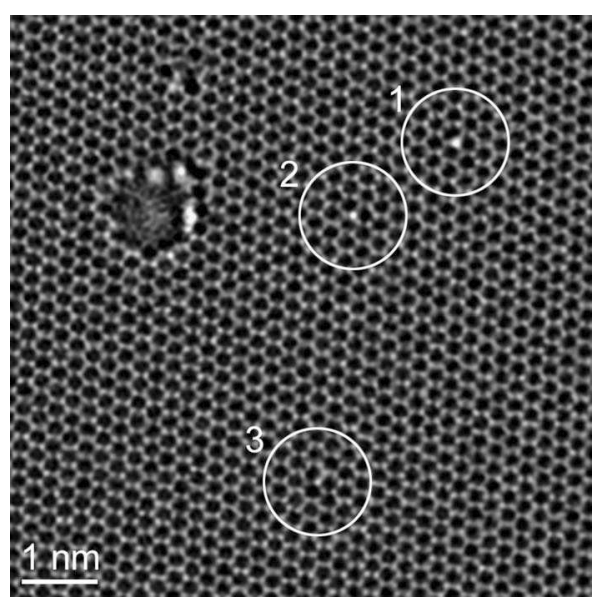

FIG. 1. Phase image of the reconstructed exit-plane wave of a monolayer graphene. The exit-plane wave was reconstructed from an 11-member focal series recorded at $80 \mathrm{kV}$.

focal series, individual micrographs and focus-invariant time series were analyzed. Focal series were recorded by applying a focus step of $-0.61 \mathrm{~nm}$. From a series containing $30 \mathrm{mem}-$ bers, 11 consecutive micrographs covering a focus range from -5.6 to $-11.7 \mathrm{~nm}$ were selected for the restoration of the exit-plane wave whose phase image is shown in Fig. 1. The third- and fifth-order spherical aberrations were corrected to $C_{3}<|1 \mu \mathrm{m}|, C_{5}<100 \mu \mathrm{m}$, and $C_{C}<20 \mu \mathrm{m}$. The stability of the defects analyzed dictates the maximum number of images that can be used for the restoration of the exit-plane wave. Yet, adequate to discuss point defects on graphene, the restorable image frequencies lie between 50 and $320 \mathrm{pm} .{ }^{21}$ With an electron dose of about $10^{6} e^{-} / \mathrm{s} \mathrm{nm}^{2}$, time series and focal series were recorded using an exposure time of $1.0 \mathrm{~s}$ per micrograph. Multislice TEM simulations of exit-plane waves were done for a resolution of $0.08 \mathrm{~nm}$.

\section{B. First-principles calculations}

TEM experiments were complemented with densityfunctional theory (DFT) calculations. These first-principles calculations were performed with the Gaussian and planewaves (GPWs) (Ref. 22) approach using the Perdew-Burke-Ernzerhof ${ }^{23}$ exchange-correlation functional and periodic boundary conditions implemented in $\mathrm{CP} 2 \mathrm{~K} .{ }^{24}$ The electron-ion interaction was described by the normconserving pseudopotentials of Goedecker-Teter-Hutter ${ }^{25}$ and for most elements a TZV2P basis set was used, optimized for molecular systems. ${ }^{26}$ The van der Waals interaction was considered using a semiempirical scheme in the form of $C_{6} / R^{6}$ proposed by Grimme. ${ }^{27}$ Diffusion barriers were calculated with GPW in its spin-polarized formulation using the climbing-image nudged elastic band method, CI-NEB. ${ }^{28}$ For each NEB calculation, 12 images are used to calculate the diffusion barrier accurately. The graphene sheet is represented by a $21.34 \times 19.71 \AA^{2}$ rectangular supercell containing 160 carbon atoms.

\section{RESULTS}

The monolayer graphene sheets as employed in this study are typically covered with thin layers of amorphous hydro- carbon contaminants which during electron irradiation are mobile and gradually disappear, eventually leaving patches of clean graphene membranes. Depending on the electron dose, this local cleaning occurs within a few minutes. During prolonged electron irradiation point defects appear ${ }^{12}$ which can expand to holes that keep on growing and can lead to the destruction of the membranes. ${ }^{16}$ During this process, part of the atoms, which are ejected by the electron beam stemming either from the contaminants or from the edges of holes, remain on the graphene. Some of these atoms might migrate fast and eventually disappear from the field of view being essentially invisible to the microscopic investigation which occurs in snapshots on the order of seconds. Yet, other atoms, not necessarily different elements, stay for an extended period of time on the graphene, might form small functional groups and are covalently or ionically attached to it. ${ }^{5}$ These are the molecular groups that are observable in the electron microscope. The configuration, stability and dynamics of such molecules were studied in the present investigation.

The phase image in Fig. 1 shows a monolayer suspended graphene containing a hole with a diameter of about $1 \mathrm{~nm}$. Above the hole, lattice defects are observable, which, similar to the edge of the hole, were subject to changes during the acquisition of the series. In addition, three pointlike "defects" are observable which are encircled. Defects 1 and 2 were invariant while the focal series was recorded [see Movie S1 (Ref. 29)].

Defects 1 and 2 show distinctly larger phase shifts on positions where one would expect single carbon atoms [see Fig. S1 (Ref. 29)]. There are two possibilities for the enhanced phase shifts: either a carbon atom of the graphene lattice is substituted by a heavier atom or an adatom is attached atop of a graphene carbon atom on a so-called $T$ site. Figure 1 does not provide a means for distinguishing between these two possibilities. However, during the acquisition of the series, the location of defect 3 changed. Figure 2 shows extracts of the micrographs recorded at defoci of $-8.04 \mathrm{~nm}$ [Figs. 2(a) and 2(c)] and $-8.65 \mathrm{~nm}$ [Figs. 2(b) and 2(d)]. Although the positions of defects 1 and 2 remain unchanged, the position of defect 3 changes: it moves from one carbon atom to a neighboring carbon position. Since a correlated diffusion-based exchange of a substitutional atom with a graphene carbon atom is unlikely to occur at room temperature without having a vacancy involved, ${ }^{30}$ we conclude that the defects in Fig. 1 are due to atoms attached to the graphene lattice as explained above and schematically illustrated in Figs. 2(e) and 2(f).

Any atom can in some way be supported by a graphene membrane. Yet, whether, where and how an atom is attached to graphene depends on the element-specific interaction. In order to narrow down the amount of potential elements, exitplane waves were simulated for a variety of adatoms (artificially) sitting on $T$ sites. In the experimental data, the ratio between the phase of the reconstructed exit-plane wave at the position of the adatoms and the phase of a carbon atom in graphene is about 1.4-1.6. The simulations show that for $\mathrm{Na}$ in the role of an adatom this ratio is about 2.0 , for $\mathrm{Li}$ it is about 1.4 and for $\mathrm{H}$ it is about 1.1 (Fig. S1). Considering the experimental fluctuation of the phase shifts of carbon atoms, which exceed $10 \%$, we conclude that the detection of single 


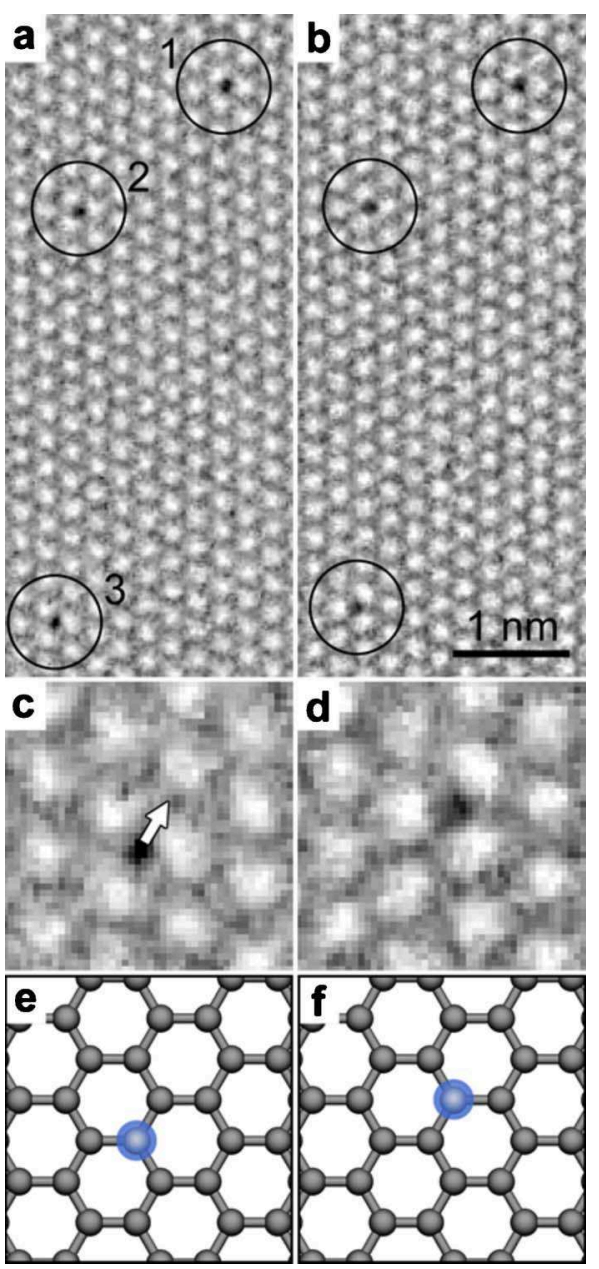

FIG. 2. (Color online) Dynamics of a $T$-site admolecule. [(a) and (b)] Extracts of two micrographs of the focal series. Atoms appear dark on a bright background (underfocus). The defects indicated in Fig. 1 appear as dark spots. While the positions of defect 1 and 2 are invariant, the position of defect 3 changes between these two snapshots, see the details of (a) and (b) shown in (c) and (d). An admolecule changes its site as schematically depicted in (e) and (f). As a result of the migration, defect 3 appears to be blurred in the time-averaged phase image in Fig. 1. See also Movie S1 (Ref. 29).

hydrogen atoms is not feasible with our data and, that the adatom has to be lighter than $\mathrm{Na}$ and heavier than Li. However, from this limited amount of potential elements, metals of group I-III (e.g., $\mathrm{Li}$ and $\mathrm{Na}$ ) find stable positions in $H$ sites, i.e., in the center of the hexagons, ${ }^{5}$ while oxygen, nitrogen, and carbon atoms attach to $B$ sites, i.e., centered above two carbon atoms. All these elements thus cannot explain the observation. Indeed, our first-principles calculations reveal that fluorine is the only element which can account for the observed phase shift and finds a stable position on a $T$ site. However, since the sample was not exposed to any source of fluorine, its presence is unlikely. On the other hand, the formation of the hole and the presence of the hydrocarbon contaminants mean that there is an abundant reservoir of carbon and hydrogen atoms present. ${ }^{31}$ Moreover, as the membranes were (unavoidably) exposed to air and various organochemical solutions during the preparation, the presence of oxygen, nitrogen, carbon, and hydrogen atoms, is

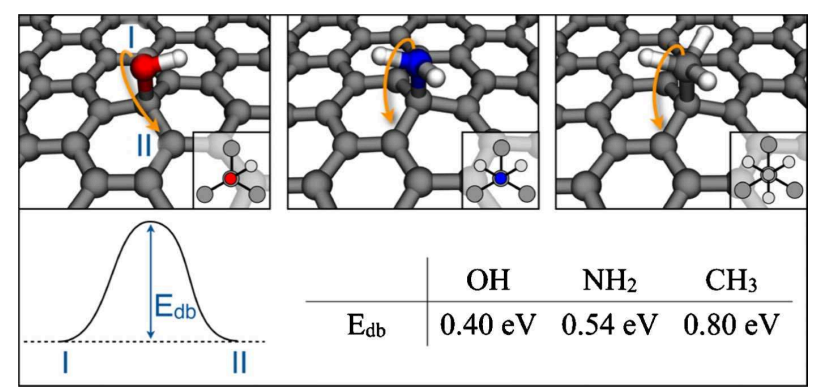

FIG. 3. (Color online) Diffusion paths and diffusion barriers $E_{\mathrm{db}}$ of $\mathrm{OH}$ (left), $\mathrm{NH}_{2}$ (middle), and $\mathrm{CH}_{3}$ (right) on graphene. The corresponding value for a hydrogen atom is $1.2 \mathrm{eV}$. C: gray, $\mathrm{H}$ : white, $\mathrm{O}$ : red, and $\mathrm{N}$ : blue.

highly probable. Yet, as all atoms likely present do not attach to $T$ sites, we conclude that single adatoms cannot explain the observation documented in Fig. 1.

For the above argument and because single carbon, nitrogen, and oxygen atoms hydrogenate in the presence of hydrogen, it is necessary to expand the picture to include small groups of atoms, such as the hydroxyl $(-\mathrm{OH})$, the amino $\left(-\mathrm{NH}_{2}\right)$, or the methyl $\left(-\mathrm{CH}_{3}\right)$ group. Our DFT calculations reveal that these groups find stable positions on $T$ sites, whereas other candidates such as $\mathrm{CO}, \mathrm{CO}_{2}, \mathrm{H}_{2} \mathrm{O}, \mathrm{NO}, \mathrm{NO}_{2}$, $\mathrm{NH}_{3}$, or $\mathrm{NH}$ do not attach to $T$ sites. ${ }^{11,32}$ Performing simulations of exit-plane waves based on atomic models derived from DFT calculations reveals that the $\mathrm{OH}, \mathrm{NH}_{2}$, and $\mathrm{CH}_{3}$ groups can explain the contrast features observed in the experimental exit-plane wave.

However, although these groups would attach to $T$ sites at zero temperature, TEM was carried out at room temperature. In agreement with previously published results, ${ }^{9}$ our calculations confirm that the migration barriers of these groups are in the range of $0.4-0.8 \mathrm{eV}$, whereas for $\mathrm{H}$ it is about $1.2 \mathrm{eV}$, see Fig. 3. This small energy difference translates into a migration probability of $\mathrm{OH}, \mathrm{NH}_{2}$, and $\mathrm{CH}_{3}$ which is more than six orders of magnitude larger than the one of $\mathrm{H}$ at room temperature. ${ }^{7}$ Hence, within a fraction of a second, one would expect the molecules to diffuse quickly on graphene or even desorb. ${ }^{9}$ Electron irradiation might even enhance their mobility. Our observation of distinct $T$-site point defects would be impossible.

\section{DISCUSSION}

The TEM observations reveal that the admolecules are stable during periods of several seconds or even minutes whereas the DFT calculations predict that isolated $\mathrm{OH}, \mathrm{NH}_{2}$, and $\mathrm{CH}_{3}$ molecules would be largely mobile on graphene at room temperature. Hence, the molecules must be stabilized by a favorable graphene configuration. Forming a single covalent bond with graphene implies that one unpaired electron is left behind in one of the benzol rings of which the graphene lattice is constituted. This unpaired electron, locally violating Hückel's rule, reduces the stability of the configuration. However, if in the immediate neighborhood an additional adatom is present as, for instance, a hydrogen atom which we are not able to observe, no unpaired electron 


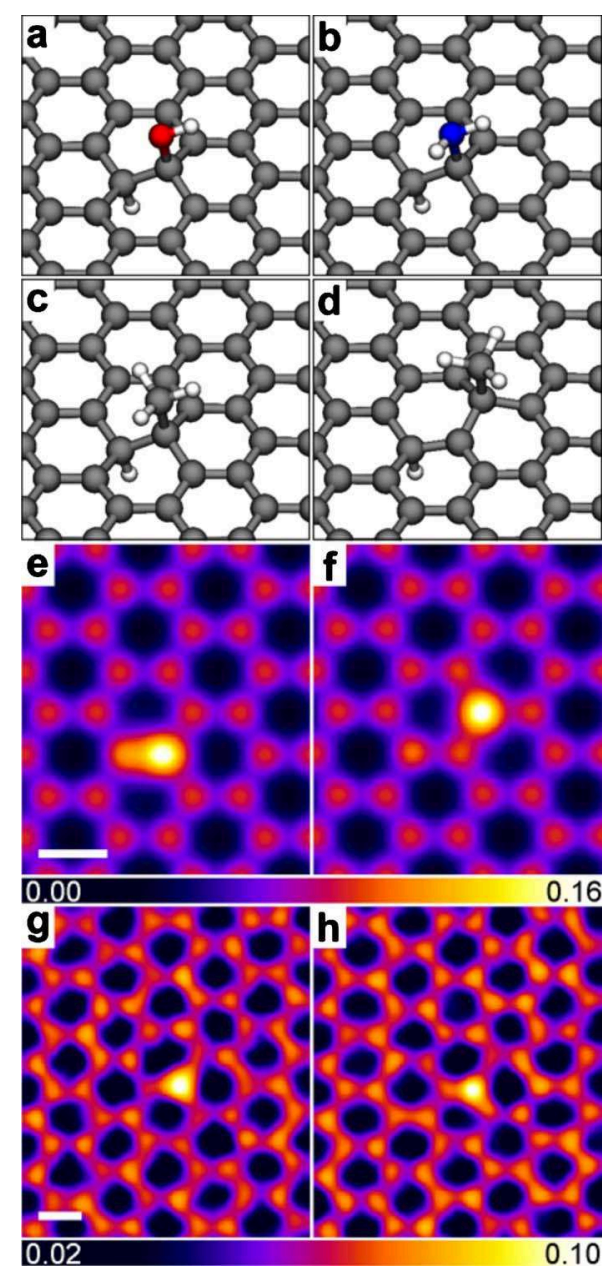

FIG. 4. (Color online) [(a)-(c)] Atomic models derived from DFT calculations. $\mathrm{OH}, \mathrm{NH}_{2}$, and $\mathrm{CH}_{3}$ are covalently bonded to graphene with the adjacent carbon atom decorated with a $\mathrm{H}$ atom. $\mathrm{C}$ : gray, $\mathrm{H}$ : white, $\mathrm{O}$ : red, and $\mathrm{N}$ : blue. (d) The $\mathrm{CH}_{3}$ group moves from the stable $T$ site to an adjacent carbon atom, which requires to surpass an energy barrier of $2.2 \mathrm{eV}$. This barrier, similar for $\mathrm{OH}$ $(1.7 \mathrm{eV})$ and $\mathrm{NH}_{2}(2.0 \mathrm{eV})$, traps the molecules in the configurations (a)-(c). [(e) and (f)] Phase images of simulated exit-plane waves for the models in (c) and (d). [(g) and (h)] Phase images of the defects 1 and 2 of Fig. 1. The scale bar in (e) and (g) is $0.2 \mathrm{~nm}$. The color bars give the phase in rad.

remains. Figure 4(a) shows an $\mathrm{OH}$ group attached to a $T$ site with the adjacent carbon atom of the graphene lattice decorated with a hydrogen atom. Figures 4(b) and 4(c) show equivalent configurations for $\mathrm{NH}_{2}$ and $\mathrm{CH}_{3}$. Similar for all three molecules, the respective bonding energy increases by $\sim 0.25 \mathrm{eV}$ if the adjacent graphene carbon atom is decorated with a hydrogen atom.

On the basis of the situations depicted in Figs. 4(a)-4(c), diffusion of the molecules on graphene can be considered. The DFT calculations reveal that the migration barrier of the $\mathrm{CH}_{3}$ group to the adjacent carbon atom in the benzol ring, as depicted in Fig. 4(d), is $2.2 \mathrm{eV}$ and, that the resulting configuration has an energy which is $1.7 \mathrm{eV}$ higher than the one depicted in Fig. 4(c). Hence, the $\mathrm{CH}_{3}$ group is trapped in the configuration shown in Fig. 4(c). Figures 4(e) and 4(f) show phase images of simulated exit-plane waves of the configu-

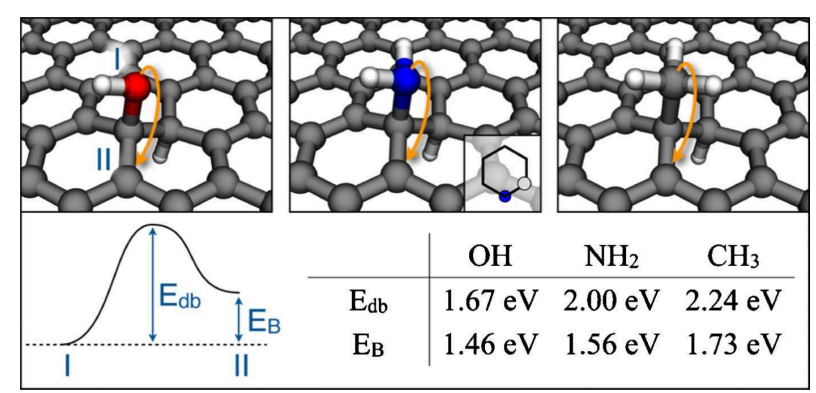

FIG. 5. (Color online) Diffusion paths, diffusion barriers $E_{\mathrm{db}}$, and binding energy $E_{\mathrm{B}}$ of the final state in respect to the original of $\mathrm{OH}$ (left), $\mathrm{NH}_{2}$ (middle), and $\mathrm{CH}_{3}$ (right) on graphene in the presence of a hydrogen atom on adjacent graphene $T$ site. $\mathrm{C}$ : gray, $\mathrm{H}$ : white, O: red, and $\mathrm{N}$ : blue.

rations depicted in Figs. 4(c) and 4(d). Hence, within the limits given by the experimental noise and resolution, the theoretical models provide feasible solutions to our observations. Carrying out similar TEM simulations for $\mathrm{OH}$ and $\mathrm{NH}_{2}$ yields exit-plane waves which are visually indistinguishable from the one of $\mathrm{CH}_{3}$. Indeed, the same trapping mechanism applies to $\mathrm{OH}$ and $\mathrm{NH}_{2}$. The diffusion paths and diffusion barriers are summarized in Fig. 5.

Trapping functional groups in the configurations depicted in Figs. 4(a)-4(c), does not mean that no dynamics takes place, particularly under electron irradiation. Our observations provide evidence that the molecules change their position every few seconds. Yet, as documented in Fig. 6, which shows three consecutive micrographs of a time series, admolecule 1 migrates to an adjacent carbon atom, but bounces back to the original position. For $\mathrm{OH}$, this is one of two equally likely events that can occur. For $\mathrm{CH}_{3}$ and $\mathrm{NH}_{2}$, the most likely event that follows the displacement shown in Fig. 6(b) is that hydrogen moves to the site the admolecule occupied previously. This alternative path is in agreement with the dynamics of admolecule 3 in Fig. 2 and Movie S1. ${ }^{29}$ There, the admolecule remains stable on the new site.

Figure 7 outlines the diffusion paths; the position of the hydrogen atom is indicated by a gray disk and the admolecule starts its diffusion path in position I. Once the admolecule migrates to position II (see, e.g., Fig. 5), it can bounce back to the original position next to the hydrogen atom, or it

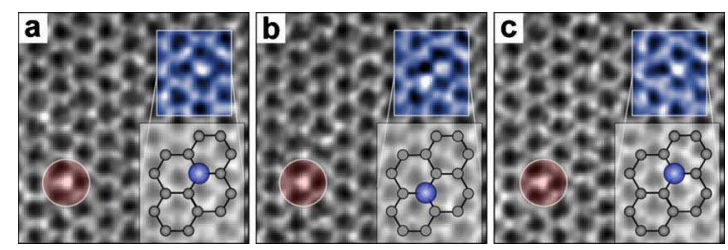

FIG. 6. (Color online) Admolecule trapped to a specific $T$ site. [(a)-(c)] Three consecutive micrographs of a time series recorded after the acquisition of the focal series (noise filtered). Here, the atoms appear bright on a dark background (overfocus). Defect 2, of Fig. 1, is indicated in red, and defect 1 is indicated in blue. (b) Admolecule 1 migrates to a neighboring carbon atom but bounces back in (c) to the original position. The insets show the corresponding models; the admolecule is indicated in blue. The distance between adjacent C atoms is $0.14 \mathrm{~nm}$. See also Movie S2 (Ref. 29). 


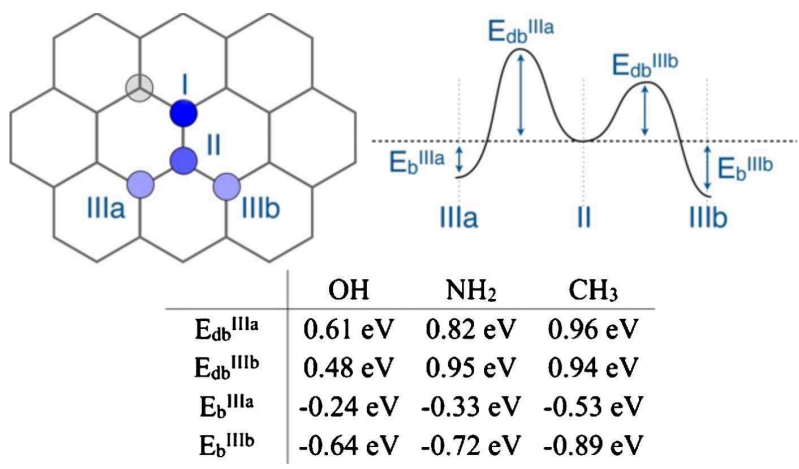

FIG. 7. (Color online) Second step in the diffusion path. Diffusion barriers of an admolecule that moved from position I to position II (see Fig. 5) to continue its path either from position II to position IIIa or IIIb. The hydrogen atom is indicated by the gray circle, next to position I.

can move on to position IIIa, i.e., stay on the same benzol ring like the hydrogen atom or, leave the benzol ring and thus move to position IIIb. The diffusion barriers to bounce back to the original position is $E_{\mathrm{db}}-E_{\mathrm{B}}$ with the values for $E_{\mathrm{db}}$ and $E_{\mathrm{B}}$ given in Fig. 5. Hence, for $\mathrm{CH}_{3}$ it is $0.51 \mathrm{eV}$, for $\mathrm{NH}_{2}$ it is $0.44 \mathrm{eV}$, and for $\mathrm{OH}$ it is $0.21 \mathrm{eV}$. Figure 7 gives the diffusion barriers $E_{\mathrm{db}}^{\mathrm{III}}$ and $E_{\mathrm{db}}^{\mathrm{III}}$ for the admolecule to move from position II to position IIIa and IIIb, respectively. For all admolecules considered here, $E_{\mathrm{db}}^{\mathrm{III}}$ and $E_{\mathrm{db}}^{\mathrm{III}}$ are larger than the diffusion barrier for moving from position II to I. Hence, under the assumption that the hydrogen atom stays in its original position, the most likely event that follows a move of the admolecule from I to II is the move back to I. This is documented in Fig. 6.

However, the assumption that the hydrogen can stay in its original position only holds if the diffusion barrier of the hydrogen atom to move from its original position to position I is similar or larger than the diffusion barrier of the admolecule to move from position II back to I (see Fig. 7). This is only the case for the $\mathrm{OH}$ group in the role of the admolecule. For the case of $\mathrm{OH}$, the diffusion barrier of the hydrogen atom to follow the admolecule to position $\mathrm{I}$ is $0.2 \mathrm{eV}$, which is similar to the diffusion barrier of $\mathrm{OH}$ to move from position II to I (see Fig. 5). This is in agreement with the dynamics documented in Fig. 6 (see also Movie S2); admolecule 1 moves from one position to a neighboring position and bounces back to the original one.

On the other hand, once $\mathrm{CH}_{3}$ or $\mathrm{NH}_{2}$ move from position I to II, it is likely that the hydrogen atom follows the admolecule to position I. The migration barrier of the hydrogen atom to follow the admolecule to position $\mathrm{I}$ is for $\mathrm{CH}_{3}$ (now on position II) $0.12 \mathrm{eV}$ and for $\mathrm{NH}_{2}$ (now on position II) $0.16 \mathrm{eV}$. Once the hydrogen atom moves to position $\mathrm{I}, \mathrm{CH}_{3}$ and $\mathrm{NH}_{2}$ are then again in a stable position, which is equivalent to the original position $\mathrm{I}$, as documented in Figs. 4(a)-4(c). This type of dynamics is in agreement with the series of images used for the focal series reconstruction (see Fig. 2 and Movie S1.) In this series of images, admolecule 3 moves to an adjacent $T$ site and remains on the new site, explaining the blurring of the reconstructed phase in Fig. 1.

Yet, it has to be emphasized that because the diffusion barriers $E_{\mathrm{db}}$ of all three admolecules to move from position I to II are between 1.7 and $2.2 \mathrm{eV}$ (see Fig. 5), the first step in the dynamics discussed needs a large activation energy which can, for instance, be supplied by high temperature or, as in our case, provided by a momentum transfer due to the electron irradiation. Hence, at ambient temperature and without electron irradiation, it is unlikely that the admolecules migrate as documented in Fig. 2 and 6. The presence of a hydrogen atom on a carbon site next to a $T$-site admolecule acts as a very effective trap.

\section{CONCLUSION}

On the basis of the experimental observation which are explained by employing DFT calculations, we conclude that hydrogen adatoms can trap molecules to specific sites on graphene that remain stable at room temperature. Indeed, what likely occurs in the process of forming these configurations is that due to their higher adhesion energy, hydrogen atoms first attach to graphene and then trigger molecules to find stable positions on adjacent $T$ sites. Provided that there are ways to attach hydrogen to graphene in a controlled manner and amount, this mechanism could be employed to functionalize graphene with specific molecular groups.

The results presented here, which are based on atomicresolution TEM and DFT calculations, provide insight into the chemistry and physics that takes place on graphene at room temperature. The proposed atomic configurations are energetically favorable: adatoms and molecules mutually trap each other in specific sites. These "symbiotic" configurations can explain our observation of small molecules on graphene at room temperature.

\section{ACKNOWLEDGMENTS}

The electron microscope employed was developed by the TEAM (Transmission Electron Aberration-corrected Microscopy) project which is supported by the U.S. DOE, Office of Science, Office of Basic Energy Sciences. The authors kindly acknowledge support by CEOS GmbH and FEI Co. M.-T.N., S.B., and D.P. acknowledge the Swiss National Supercomputing Centre (CSCS), and for financial support the Alexander von Humboldt Foundation and the Swiss National Science Foundation. 
*rolf.erni@empa.ch

${ }^{1}$ K. S. Novoselov, A. K. Geim, S. V. Morozov, D. Jiang, M. I. Katsnelson, I. V. Grigorieva, S. V. Dubonos, and A. A. Firsov, Nature (London) 438, 197 (2005).

${ }^{2}$ A. K. Geim and K. S. Novoselov, Nature Mater. 6, 183 (2007).

${ }^{3}$ A. K. Geim, Science 324, 1530 (2009).

${ }^{4}$ P. O. Lehtinen, A. S. Foster, A. Ayuela, A. Krasheninnikov, K. Nordlund, and R. M. Nieminen, Phys. Rev. Lett. 91, 017202 (2003).

${ }^{5}$ K. T. Chan, J. B. Neaton, and M. L. Cohen, Phys. Rev. B 77, 235430 (2008).

${ }^{6}$ X. Wang, X. Li, L. Zhang, Y. Yoon, P. K. Weber, H. Wang, J. Guo, and H. Dai, Science 324, 768 (2009).

${ }^{7}$ C. Coletti, C. Riedl, D. S. Lee, B. Krauss, L. Patthey, K. von Klitzing, J. H. Smet, and U. Starke, Phys. Rev. B 81, 235401 (2010).

${ }^{8}$ O. C. Compton and S. B. T. Nguyen, Small 6, 711 (2010).

${ }^{9}$ T. O. Wehling, M. I. Katsnelson, and A. I. Lichtenstein, Phys. Rev. B 80, 085428 (2009).

${ }^{10}$ T. Hashi, Y. Uramoto, and M. Saito, Jpn. J. Appl. Phys. 47, 6623 (2008).

${ }^{11}$ O. Leenaerts, B. Partoens, and F. M. Peeters, Phys. Rev. B 77, 125416 (2008).

${ }^{12}$ J. C. Meyer, C. Kisielowski, R. Erni, M. D. Rossell, M. F. Crommie, and A. Zettl, Nano Lett. 8, 3582 (2008).

${ }^{13}$ J. C. Meyer, Ç. Ö. Girit, M. F. Crommie, and A. Zettl, Appl. Phys. Lett. 92, 123110 (2008).

${ }^{14}$ B. Kabius, P. Hartel, M. Haider, H. Müller, S. Uhlemann, U. Loebau, J. Zach, and H. Rose, J. Electron Microsc. 58, 147 (2009).

${ }^{15}$ J. Zach, Philos. Trans. R. Soc. London, Ser. A 367, 3699 (2009).

${ }^{16}$ Ç. Ö. Girit, J. C. Meyer, R. Erni, M. D. Rossell, C. Kisielowski, L. Yang, C. H. Park, M. F. Crommie, M. L. Cohen, S. G. Louie, and A. Zettl, Science 323, 1705 (2009).

${ }^{17}$ W. Coene, G. Janssen, M. Op de Beeck, and D. Van Dyck, Phys. Rev. Lett. 69, 3743 (1992).

${ }^{18}$ M. D. Rossell, R. Erni, M. Asta, V. Radmilovic, and U. Dahmen, Phys. Rev. B 80, 024110 (2009).

${ }^{19}$ N. Alem, R. Erni, C. Kisielowski, M. D. Rossell, W. Gannett, and A. Zettl, Phys. Rev. B 80, 155425 (2009).

${ }^{20}$ R. Erni, M. D. Rossell, and P. N. H. Nakashima, Ultramicroscopy 110, 151 (2010).

${ }^{21}$ A. Thust, W. M. J. Coene, M. Op de Beeck, and D. Van Dyck, Ultramicroscopy 64, 211 (1996).

${ }^{22}$ J. VandeVondele, M. Krack, F. Mohamed, M. Parrinello, T. Chassaing, and J. Hutter, Comput. Phys. Commun. 167, 103 (2005).

${ }^{23}$ J. P. Perdew, K. Burke, and M. Ernzerhof, Phys. Rev. Lett. 78, 1396 (1997).

${ }^{24} \mathrm{CP} 2 \mathrm{~K}$, http://cp2k.berlios.de

${ }^{25}$ S. Goedecker, M. Teter, and J. Hutter, Phys. Rev. B 54, 1703 (1996).

${ }^{26}$ J. VandeVondele and J. Hutter, J. Chem. Phys. 127, 114105 (2007).

${ }^{27}$ S. Grimme, J. Comput. Chem. 27, 1787 (2006).

${ }^{28}$ G. Henkelman, B. P. Uberuaga, and H. Jonsson, J. Chem. Phys. 113, 9901 (2000).

${ }^{29}$ See supplementary material at http://link.aps.org/supplemental/ 10.1103/PhysRevB.82.165443 for additional experimental and computational details and for supporting figures and movies.

${ }^{30}$ A. V. Krasheninnikov, P. O. Lehtinen, A. S. Foster, P. Pyykkö, and R. M. Nieminen, Phys. Rev. Lett. 102, 126807 (2009).

${ }^{31}$ J. C. Meyer, Ç. Ö. Girit, M. F. Crommie, and A. Zettl, Nature (London) 454, 319 (2008).

${ }^{32}$ Z. M. Ao, J. Yang, S. Li, and Q. Jiang, Chem. Phys. Lett. 461, 276 (2008). 